\title{
Design of Adaptive Block Backstepping Controllers for Semi-strict Feedback Nonlinear Systems with Perturbation Estimation*
}

\author{
Chih-Chiang Cheng ${ }^{1}$, An-Fu Chien ${ }^{2}$ and Yi-Chun Huang ${ }^{2}$
}

\begin{abstract}
Based on the Lyapunov stability theorem, a design methodology of adaptive block backstepping control with perturbation estimation scheme is proposed in this paper for a class of multi-input systems with perturbations to solve regulation problems. First of all, the virtual input controllers are designed from the first block to the $(m-1) t h$ block. Then the proposed robust controller and the perturbation estimator are designed in accordance with the last block. Adaptive mechanisms are employed in each of the virtual input as well as the robust controller so that they may automatically adapt the least upper bounds of perturbations or perturbation estimation errors which may not fulfill the strict-feedback form. Furthermore, the proposed control scheme is able to achieve asymptotic stability. A numerical example is also given for demonstrating the feasibility of the proposed control scheme.
\end{abstract}

\section{INTRODUCTION}

In most practical control applications, the dynamic equations of plants are nonlinear and contain uncertain factors, such as model uncertainties, external disturbances etc. Therefore the robust control methods such as sliding mode control (SMC) and backstepping control have become two of the commonly used nonlinear control methods for solving regulation or tracking problems in recent years. SMC is capable of providing robustness and invariance to matched external disturbances and model uncertainties [1], [2]. If a sliding mode controller is properly designed, asymptotic stability for systems with mismatched perturbations still can be obtained [3]-[6]. However, it is observed that designing novel sliding surface functions to achieve asymptotic stability for mismatched perturbed systems are in general not easy and quite complicated.

On the other hand, for nonlinear systems with dynamic equations in the so called "strict feedback form", both feedback controllers and associated Lyapunov functions can be constructed systematically by using backstepping control technique [7], [8]. The main design procedure of backstepping controller involves assigning of new state variables and design of virtual input controls, which is fairly flexible, and is designed in accordance with control's objectives. The main advantage of this method is that it is quite effective

\footnotetext{
*This work was supported in part by the R. O. C. National Science Council under Grants NSC 100-2221-E-110-025, NSC 102-3113-P-110-005

${ }^{1}$ C.-C. Cheng is with Faculty of Electrical Engineering, National Sun Yat-Sen University, 804 Kaohsiung, Taiwan, R.O.C. chengcc@mail.ee.nsysu.edu.tw

${ }^{2}$ A.-F. Chien is with the Department of Electrical Engineering, National Sun Yat-Sen University, 804 Kaohsiung, Taiwan, R.O.C. m973010140@student.nsysu.edu.tw

${ }^{2}$ Y.-C. Huang is with the Department of Electrical Engineering, National Sun Yat-Sen University, 804 Kaohsiung, Taiwan, R.O.C. m993010132@student.nsysu.edu.tw
}

to deal with systems which contain matched or mismatched perturbations in order to achieve the property of asymptotic stability [7]. Another advantage is that it is a recursive procedure that keeps repeating the same design steps.

For controlling MIMO systems by using backstepping technique, it is found that the systems to be controlled in [9]-[13] have to be in strict-feedback form, which is known as a major disadvantage of backstepping control method. Although the dynamic equations of the plants to be controlled in [14]-[17] are more general than those with strict feedback form, but the upper bounds of perturbations encountered in the systems are still required to be in strictfeedback form. The adaptive block backstepping control schemes presented in [18]-[21] were also for alleviating the constraint of strict-feedback form, but block strict-feedback form are still required. Some researchers [22]-[24] combined backstepping with fuzzy (or neural) and dynamic surface control (DSC) method in order to alleviate the phenomenon of "explosion of complexity", which is also known as a limitation of backstepping method. However, it is found that only UUB can be achieved and the initial condition of the systems may not be arbitrarily assigned when DSC method is applied in the design of backstepping control systems.

Due to the limitations of each of the methodologies mentioned above, in this paper we propose a design methodology of adaptive block backstepping control with perturbation estimation scheme for a class of perturbed multi-input systems to solve regulation problems. The dynamic equations of the systems need not satisfy the block-strict feedback form. The design procedure of the proposed controllers mainly involves two steps. The first step is the design of virtual input controllers from the first block to the $(m-1) t h$ block. The second step is the design of robust controller and adaptive gains as well as perturbation estimator in accordance with the $m t h$ block to guarantee asymptotic stability of whole controlled system. The upper bounds of perturbations and perturbation estimation errors are not required to be known in advance due to the embedded adaptive mechanisms, and the control energy is in general reduced due to the usage of perturbation estimator. Finally, the proposed method is applied to a numerical example for demonstrating the feasibility of the proposed control scheme.

\section{Design of AdAPtive BackstepPing CONTROllers WITH PERTURBATION ESTIMATION}

\section{A. System Descriptions and Problem Formulations}

Consider a class of multi-input plants with both matched and mismatched perturbations. The dynamic equations of 
these plants are governed by

$$
\begin{aligned}
\dot{\mathbf{x}}_{i} & =\mathbf{f}_{i}\left(t, \overline{\mathbf{x}}_{i}\right)+\mathbf{G}_{i}\left(\overline{\mathbf{x}}_{i}\right) \mathbf{x}_{i+1}+\triangle \mathbf{f}_{i}(t, \mathbf{x}), \\
\dot{\mathbf{x}}_{m} & =\mathbf{f}_{m}(t, \mathbf{x})+\mathbf{G}_{m}(\mathbf{x}) \mathbf{u}+\Delta \mathbf{f}_{m}(t, \mathbf{x}),
\end{aligned}
$$

for $1 \leq i \leq m-1$, where $\mathbf{x}=\left[\begin{array}{llll}\mathbf{x}_{1}^{T} & \mathbf{x}_{2}^{T} & \cdots & \mathbf{x}_{m}^{T}\end{array}\right]^{T}$ represents measurable state vector, $\overline{\mathbf{x}}_{j}=\left[\begin{array}{llll}\mathbf{x}_{1}^{T} & \mathbf{x}_{2}^{T} & \cdots & \mathbf{x}_{j}^{T}\end{array}\right]^{T}, 1 \leq j \leq m$, $m \geq 2, \mathbf{x}_{\ell} \in R^{n_{\ell}}$, and $n_{\ell} \leq n_{\ell+1}, \quad 1 \leq \ell \leq m-1$. The nonlinear vectors $\mathbf{f}_{j}\left(t, \overline{\mathbf{x}}_{j}\right) \in R^{n_{j}}, 1 \leq j \leq m$, are known and $\mathbf{f}_{j}\left(t, \overline{\mathbf{x}}_{j}\right)=\mathbf{0}$ if $\overline{\mathbf{x}}_{j}=\mathbf{0}$. The matrices $\mathbf{G}_{i} \in R^{n_{i} \times n_{i+1}}, \mathbf{G}_{m} \in R^{n_{m} \times n_{m}}, \quad 1 \leq i \leq m-1$, are also known, and $\mathbf{u} \in R^{n_{m}}$ is the control input. The vectors $\triangle \mathbf{f}_{j}(\bullet) \in R^{n_{j}}$ can be viewed as unknown nonlinearities, model uncertainties or external disturbances. Noted that (1) is not in strict-feedback form due to the perturbations $\Delta \mathbf{f}_{j}(\bullet)$.

In order to regulate the response of dynamic system (1) in spite of the existence of perturbations, we assume that the following assumptions are valid throughout this paper:

A1: The matrices $\mathbf{G}_{j}\left(\overline{\mathbf{x}}_{j}\right), 1 \leq j \leq m$, have full rank $n_{j}$ for all $\overline{\mathbf{x}}_{j}$ in the domain of interest.

A2: The vectors $\mathbf{f}_{i}\left(t, \overline{\mathbf{x}}_{i}\right)$ and matrices $\overline{\mathbf{G}}_{i} \triangleq \mathbf{G}_{i}^{T}\left(\mathbf{G}_{i} \mathbf{G}_{i}^{T}\right)^{-1}$ are continuously differentiable with respect to $\overline{\mathbf{x}}_{i}, 1 \leq i \leq$ $m-1$.

A3: $\triangle \mathbf{f}_{i}(\bullet)=\mathbf{0}$ if $\mathbf{x}=\mathbf{0}, 1 \leq i \leq m-1$, and the following inequalities

$$
\begin{aligned}
\left\|\triangle \mathbf{f}_{i}(t, \mathbf{x})\right\| & \leq \sum_{\ell=1}^{m} b_{i \ell}\left\|\mathbf{x}_{\ell}\right\|, \quad 1 \leq i \leq m-1 \\
\left\|\triangle \tilde{\mathbf{f}}_{m}(t, \mathbf{x})\right\| & \leq \beta_{0}+\beta_{m}\|\mathbf{x}\|
\end{aligned}
$$

are fulfilled in the domain of interest, where $b_{i \ell}, \beta_{0}$, and $\beta_{m}$ are unknown positive constants. The vector $\triangle \tilde{\mathbf{f}}_{m} \triangleq$ $\triangle \mathbf{f}_{m}-\dot{\alpha}_{(m-1) p}-\mathbf{p}_{\text {est }}(t)$ is the perturbation estimation error, and $\mathbf{p}_{e s t}(t)$ is the estimation of the signal $\triangle \mathbf{f}_{m}-\dot{\alpha}_{(m-1) p}$. The signal $\dot{\alpha}_{i n}$ denotes the nominal part of the derivative of virtual inputs $\boldsymbol{\alpha}_{i}, 1 \leq i \leq m-1$, and $\dot{\boldsymbol{\alpha}}_{i p}$ is the part of $\dot{\boldsymbol{\alpha}}_{i}$ which contains perturbations (Appendix C).

In this paper, the notation $\|\mathbf{M}\|$ denotes the induced 2norm of a matrix $\mathbf{M}$, and $\|\mathbf{x}\|$ stands for Euclidean norm of a vector $\mathbf{x}$. The main objective of this paper is to develop a control algorithm using backstepping method as well as perturbation estimation so that the state trajectory $\mathbf{x}(t)$ of perturbed dynamic equation (1) can achieve the property of asymptotic stability.

\section{B. Design of the Controllers}

Although (1) is not in strict-feedback form, the traditional backstepping control technique is still applicable when designing the proposed controller for system (1). The problem one needs to solve is how to handle the terms which are not in strict-feedback form in (1). According to the backstepping methodology, one should design virtual inputs $\boldsymbol{\alpha}_{i}, 1 \leq i \leq$ $m-1$, first. We first define new state variables $\mathbf{z}_{j}$ as

$$
\mathbf{z}_{j}\left(\overline{\mathbf{x}}_{j}\right) \triangleq \mathbf{x}_{j}-\boldsymbol{\alpha}_{j-1}\left(\overline{\mathbf{x}}_{j-1}\right), \quad 1 \leq j \leq m
$$

where $\boldsymbol{\alpha}_{0} \triangleq \mathbf{0}$. The details of designing these virtual inputs $\boldsymbol{\alpha}_{i}$ are demonstrated in the following steps.

Step 1 :
From (2), one is able to see that the dynamics of $\mathbf{x}_{1}$ is equivalent to

$$
\dot{\mathbf{x}}_{1}=\mathbf{f}_{1}+\mathbf{G}_{1} \boldsymbol{\alpha}_{1}+\triangle \mathbf{f}_{1}+\mathbf{G}_{1} \mathbf{z}_{2} .
$$

Now define a Lyapunov function candidate as $V_{1} \triangleq \mathbf{z}_{1}^{T} \mathbf{z}_{1} / 2$. By using the result of Appendix A, one is able to obtain the time derivative of $V_{1}$ along the trajectory of (3) as

$$
\begin{aligned}
\dot{V}_{1} & =\mathbf{z}_{1}^{T}\left[\mathbf{f}_{1}+\mathbf{G}_{1} \boldsymbol{\alpha}_{1}+\triangle \mathbf{f}_{1}+\mathbf{G}_{1} \mathbf{z}_{2}\right] \\
& \leq \mathbf{z}_{1}^{T}\left[\mathbf{f}_{1}+\mathbf{G}_{1} \boldsymbol{\alpha}_{1}\right]+\mathbf{z}_{1}^{T} \mathbf{G}_{1} \mathbf{z}_{2}+\sum_{\ell=1}^{m} \bar{r}_{1 \ell}\left\|\mathbf{z}_{\ell}\right\|^{2} .
\end{aligned}
$$

Since the matrices $\mathbf{G}_{i}\left(\overline{\mathbf{x}}_{i}\right)$ have full rank $n_{i},\left(\mathbf{G}_{i} \mathbf{G}_{i}^{T}\right)^{-1}$ exist $1 \leq i \leq m-1$. One can design the virtual input controller $\alpha_{1}$ as

$$
\boldsymbol{\alpha}_{1}\left(\overline{\mathbf{x}}_{1}\right)=-\overline{\mathbf{G}}_{1}\left[\mathbf{f}_{1}+\left(\varepsilon_{1}+\hat{\theta}_{1}(t)\right) \mathbf{z}_{1}\right]
$$

where $\varepsilon_{1}$ is a designed positive constant, and the adaptive gain $\hat{\theta}_{1}(t)$ will be determined later. Substituting (5) into (4) yields

$$
\dot{V}_{1} \leq-\left(\varepsilon_{1}+\hat{\theta}_{1}\right)\left\|\mathbf{z}_{1}\right\|^{2}+\mathbf{z}_{1}^{T} \mathbf{G}_{1} \mathbf{z}_{2}+\sum_{\ell=1}^{m} \bar{r}_{1 \ell}\left\|\mathbf{z}_{\ell}\right\|^{2} .
$$

\section{Step 2 :}

In this step, one is able to see that the dynamics of $\mathbf{x}_{2}$ is equivalent to

$$
\dot{\mathbf{x}}_{2}=\mathbf{f}_{2}+\mathbf{G}_{2} \boldsymbol{\alpha}_{2}+\triangle \mathbf{f}_{2}+\mathbf{G}_{2} \mathbf{z}_{3} .
$$

Define the second Lyapunov function candidate as $V_{2} \triangleq$ $\mathbf{z}_{2}^{T} \mathbf{z}_{2} / 2+V_{1}$. By using (6) and the result of Appendix B, one can compute the time derivative of $V_{2}$ along the trajectory of (7) as

$$
\begin{aligned}
\dot{V}_{2} \leq & -\left(\varepsilon_{1}+\hat{\theta}_{1}\right)\left\|\mathbf{z}_{1}\right\|^{2}+\mathbf{z}_{2}^{T}\left[\mathbf{G}_{1}^{T} \mathbf{z}_{1}+\mathbf{f}_{2}+\mathbf{G}_{2} \boldsymbol{\alpha}_{2}-\dot{\boldsymbol{\alpha}}_{1 n}\right] \\
& +\mathbf{z}_{2}^{T} \mathbf{G}_{2} \mathbf{z}_{3}+\sum_{q=1}^{2} \sum_{\ell=1}^{m} \bar{r}_{q \ell}\left\|\mathbf{z}_{\ell}\right\|^{2} .
\end{aligned}
$$

Now one can design the virtual input controller $\boldsymbol{\alpha}_{2}$ as

$$
\boldsymbol{\alpha}_{2}\left(\overline{\mathbf{x}}_{2}\right)=-\overline{\mathbf{G}}_{2}\left[\mathbf{G}_{1}^{T} \mathbf{z}_{1}+\mathbf{f}_{2}+\left(\varepsilon_{2}+\hat{\theta}_{2}(t)\right) \mathbf{z}_{2}-\dot{\boldsymbol{\alpha}}_{1 n}\right],
$$

where $\varepsilon_{2}$ is a designed positive constant, and the adaptive gain $\hat{\theta}_{2}(t)$ will be determined later. Substituting (9) into (8) yields

$$
\dot{V}_{2} \leq-\sum_{p=1}^{2}\left(\varepsilon_{p}+\hat{\theta}_{p}\right)\left\|\mathbf{z}_{p}\right\|^{2}+\mathbf{z}_{2}^{T} \mathbf{G}_{2} \mathbf{z}_{3}+\sum_{q=1}^{2} \sum_{\ell=1}^{m} \bar{r}_{q \ell}\left\|\mathbf{z}_{\ell}\right\|^{2} .
$$

Step $i: i=3 \sim m-1$

In this step one is able to see that the dynamics of $\mathbf{x}_{i}$ is equivalent to

$$
\dot{\mathbf{x}}_{i}=\mathbf{f}_{i}+\mathbf{G}_{i} \boldsymbol{\alpha}_{i}+\triangle \mathbf{f}_{i}+\mathbf{G}_{i} \mathbf{z}_{i+1} .
$$

According to (6) and (10), it is reasonable to assume that

$$
\dot{V}_{i-1} \leq-\sum_{p=1}^{i-1}\left(\varepsilon_{p}+\hat{\theta}_{p}\right)\left\|\mathbf{z}_{p}\right\|^{2}+\mathbf{z}_{i-1}^{T} \mathbf{G}_{i-1} \mathbf{z}_{i}+\sum_{q=1}^{i-1} \sum_{\ell=1}^{m} \bar{r}_{q \ell}\left\|\mathbf{z}_{\ell}\right\|^{2} .
$$


Now define the $i$ th Lyapunov function candidate as

$$
V_{i} \triangleq \frac{1}{2} \mathbf{z}_{i}^{T} \mathbf{z}_{i}+V_{i-1}
$$

By using (12) and the result of Appendix B, one can compute the time derivative of $V_{i}$ along the trajectory of (11) as

$$
\begin{aligned}
\dot{V}_{i} \leq & -\sum_{p=1}^{i-1}\left(\varepsilon_{p}+\hat{\theta}_{p}\right)\left\|\mathbf{z}_{p}\right\|^{2}+\mathbf{z}_{i}^{T}\left[\mathbf{G}_{i-1}^{T} \mathbf{z}_{i-1}+\mathbf{f}_{i}+\mathbf{G}_{i} \boldsymbol{\alpha}_{i}\right. \\
& \left.-\dot{\alpha}_{(i-1) n}\right]+\mathbf{z}_{i}^{T} \mathbf{G}_{i} \mathbf{z}_{i+1}+\sum_{j=1}^{i} \sum_{\ell=1}^{m} \bar{r}_{j \ell}\left\|\mathbf{z}_{\ell}\right\|^{2} .
\end{aligned}
$$

According to (14), one can design the virtual input controller $\boldsymbol{\alpha}_{i}$ as

$$
\boldsymbol{\alpha}_{i}\left(\overline{\mathbf{x}}_{i}\right)=-\overline{\mathbf{G}}_{i}\left[\mathbf{G}_{i-1}^{T} \mathbf{z}_{i-1}+\mathbf{f}_{i}+\left(\varepsilon_{i}+\hat{\theta}_{i}\right) \mathbf{z}_{i}-\dot{\boldsymbol{\alpha}}_{(i-1) n}\right],
$$

where $\varepsilon_{i}$ is a designed positive constant, and the adaptive gain $\hat{\theta}_{i}(t)$ will be determined later. Substituting (15) into (14) yields

$$
\dot{V}_{i} \leq-\sum_{j=1}^{i}\left(\varepsilon_{j}+\hat{\theta}_{j}\right)\left\|\mathbf{z}_{j}\right\|^{2}+\mathbf{z}_{i}^{T} \mathbf{G}_{i} \mathbf{z}_{i+1}+\sum_{j=1}^{i} \sum_{\ell=1}^{m} \bar{r}_{j \ell}\left\|\mathbf{z}_{\ell}\right\|^{2} .
$$

Equation (16) verifies that the assumption of inequality (12) is correct from the mathematic induction point of view.

Remark 1: In fact the range of index $i$ in this step is also valid for $1 \leq i \leq m-1$.

Remark 2: The virtual input designed in the step $i$ is not able to make the Lyapunov $\dot{V}_{i}$ a negative (semi)definite function since we are dealing with systems with semi-strict feedback form, this is different from those designed in [18][20] for systems with strict-feedback form.

\section{Step $m$ :}

This final step is to design a control input $\mathbf{u}$ so that the state variable $\mathbf{x}$ can achieve the property of asymptotic stability. The proposed controller is designed as

$$
\mathbf{u}=\mathbf{u}_{e q}+\mathbf{u}_{s}
$$

where

$$
\begin{aligned}
\mathbf{u}_{e q}= & -\left(\mathbf{G}_{m}\right)^{-1}\left[\mathbf{f}_{m}+\mathbf{G}_{m-1}^{T} \mathbf{z}_{m-1}-\dot{\boldsymbol{\alpha}}_{(m-1) n}+\mathbf{p}_{e s t}\right. \\
& \left.+\left(\hat{\theta}_{m}+\epsilon_{m}\right) \mathbf{z}_{m}\right], \\
\mathbf{u}_{s}= & \begin{cases}-\left(\mathbf{G}_{m}\right)^{-1}\left[\hat{\beta}_{0}+\hat{\beta}_{m}\|\mathbf{x}\|\right] \frac{\mathbf{z}_{m}}{\left\|\mathbf{z}_{m}\right\|}, & \text { if } \mathbf{z}_{m} \neq \mathbf{0} \\
\mathbf{0}, & \text { if } \mathbf{z}_{m}=\mathbf{0}\end{cases}
\end{aligned}
$$

The adaptive gains $\hat{\theta}_{j}, 1 \leq j \leq m, \hat{\beta}_{0}$, and $\hat{\beta}_{m}$ are given by

$$
\begin{aligned}
& \dot{\hat{\theta}}_{j}(t)=\left\|\mathbf{z}_{j}\right\|^{2}, \quad 1 \leq j \leq m, \\
& \dot{\hat{\beta}}_{0}(t)=\left\|\mathbf{z}_{m}\right\|, \quad \dot{\hat{\beta}}_{m}(t)=\left\|\mathbf{z}_{m}\right\|\|\mathbf{x}\| .
\end{aligned}
$$

\section{Perturbation Estimation}

The perturbation estimation method proposed in [25] is utilized in this paper to design the signal $\mathbf{p}_{\text {est }}$. From (1), (2), and (17), it is seen

$$
\dot{\mathbf{z}}_{m}=\left\{\begin{array}{l}
-\mathbf{G}_{m-1}^{T} \mathbf{z}_{m-1}-\left(\hat{\theta}_{m}+\epsilon_{m}\right) \mathbf{z}_{m}-\mathbf{p}_{e s t}+\Delta \mathbf{f}_{m} \\
-\left(\hat{\beta}_{0}+\hat{\beta}_{m}\|\mathbf{x}\|\right) \frac{\mathbf{z}_{m}}{\left\|\mathbf{z}_{m}\right\|}-\dot{\alpha}_{(m-1) p}, \text { if } \mathbf{z}_{m} \neq \mathbf{0} \\
-\mathbf{G}_{m-1}^{T} \mathbf{z}_{m-1}-\mathbf{p}_{e s t}+\triangle \mathbf{f}_{m}-\dot{\alpha}_{(m-1) p}, \text { if } \mathbf{z}_{m}=\mathbf{0}
\end{array}\right.
$$

which also indicates that

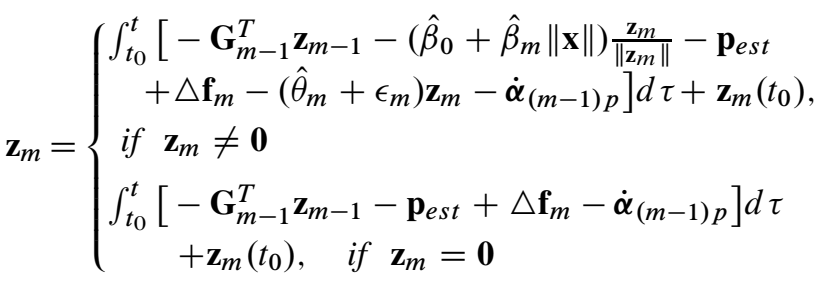

Now let two measurable signals $\overline{\mathbf{z}}_{m}$ and $\triangle \mathbf{I}(t)$ be

$\overline{\mathbf{z}}_{m} \triangleq\left\{\begin{array}{c}\int_{t_{0}}^{t}\left[-\mathbf{G}_{m-1}^{T} \mathbf{z}_{m-1}-\left(\hat{\beta}_{0}+\hat{\beta}_{m}\|\mathbf{x}\|\right) \frac{\mathbf{z}_{m}}{\left\|\mathbf{z}_{m}\right\|}\right. \\ \left.-\left(\hat{\theta}_{m}+\epsilon_{m}\right) \mathbf{z}_{m}-\mathbf{p}_{e s t}\right] d \tau+\overline{\mathbf{z}}_{m}\left(t_{0}\right), \text { if } \mathbf{z}_{m} \neq \mathbf{0} \\ \int_{t_{0}}^{t}\left[-\mathbf{G}_{m-1}^{T} \mathbf{z}_{m-1}-\mathbf{p}_{\text {est }}\right] d \tau+\overline{\mathbf{z}}_{m}\left(t_{0}\right), \text { if } \mathbf{z}_{m}=\mathbf{0}\end{array}\right.$

and

$$
\begin{aligned}
\Delta \mathbf{I}(t) & \triangleq \mathbf{z}_{m}-\overline{\mathbf{z}}_{m} \\
& =\int_{t_{0}}^{t}\left[\triangle \mathbf{f}_{m}-\dot{\alpha}_{(m-1) p}\right] d \tau+\mathbf{z}_{m}\left(t_{0}\right)-\overline{\mathbf{z}}_{m}\left(t_{0}\right),
\end{aligned}
$$

respectively. Equation (19) indicates that $d[\Delta \mathbf{I}(t)] / d t=$ $\triangle \mathbf{f}_{m}-\dot{\alpha}_{(m-1) p}$. Therefore, one simple way to estimate the perturbation $\triangle \mathbf{f}_{m}-\dot{\alpha}_{(m-1) p}$ is to compute the derivative of $\triangle \mathbf{I}(t)$, i.e.,

$$
\mathbf{p}_{e s t}=\frac{d}{d t}[\triangle \mathbf{I}(t)]=\frac{d}{d t}\left[\mathbf{z}_{m}-\overline{\mathbf{z}}_{m}\right] .
$$

The derivative estimation method proposed in [26] is utilized to estimate $\frac{d}{d t}[\triangle \mathbf{I}(t)]$.

\section{Stability AnAlysis}

Theorem 1: Consider the dynamic equations (1) with assumptions A1-A3. Suppose that the virtual inputs $\boldsymbol{\alpha}_{i}$ and $\dot{\boldsymbol{\alpha}}_{i p}$ in the domain of interest satisfy the following constraints

$$
\left\|\boldsymbol{\alpha}_{i}\left(\overline{\mathbf{x}}_{i}\right)\right\| \leq \sum_{q=1}^{i} a_{i q}\left\|\mathbf{x}_{q}\right\|, \quad\left\|\dot{\boldsymbol{\alpha}}_{i p}(\mathbf{x})\right\| \leq \sum_{\ell=1}^{m} d_{i \ell}\left\|\mathbf{x}_{\ell}\right\|,
$$

where $a_{i q}$ and $d_{i \ell}, 1 \leq q \leq i, 1 \leq \ell \leq m, 1 \leq i \leq m-1$, are unknown positive constants. If the virtual inputs and the controller are designed as (15) and (17), respectively; then (a) the trajectories of states $\mathbf{z}$ and $\mathbf{x}$ will reach zero asymptotically, and the adaptive gains $\hat{\theta}_{j}, 1 \leq j \leq m, \hat{\beta}_{0}$, and $\hat{\beta}_{m}$ are all bounded;

(b) there exist finite constants $\theta_{j \infty}, \beta_{0 \infty}$, and $\beta_{m \infty}$ such that $\lim _{t \rightarrow \infty} \hat{\theta}_{i}(t)=\theta_{i \infty}, 1 \leq i \leq m-1, \lim _{t \rightarrow \infty} \hat{\beta}_{0}(t)=\beta_{0 \infty}$, and $\lim _{t \rightarrow \infty} \hat{\beta}_{m}(t)=\beta_{m \infty}$;

(c) the stability of overall controlled system is guaranteed. 
Proof: (a) Define a Lyapunov function candidate as

$$
V_{m}=V_{m-1}+\frac{1}{2} \mathbf{z}_{m}^{T} \mathbf{z}_{m}+\frac{1}{2} \sum_{\ell=1}^{m} \tilde{\theta}_{\ell}^{2}+\frac{1}{2} \tilde{\beta}_{0}^{2}+\frac{1}{2} \tilde{\beta}_{m}^{2},
$$

where $\tilde{\theta}_{\ell} \triangleq \theta_{\ell}-\hat{\theta}_{\ell}, 1 \leq \ell \leq m, \tilde{\beta}_{0} \triangleq \beta_{0}-\hat{\beta}_{0}$, and $\tilde{\beta}_{m} \triangleq \beta_{m}-\hat{\beta}_{m}$ are the adaptive errors of the unknown positive constants $\theta_{\ell} \triangleq \sum_{j=1}^{m-1} \bar{r}_{j \ell}, \beta_{0}$, and $\beta_{m}$ respectively. By noting that $\dot{\tilde{\theta}}_{\ell}=-\dot{\hat{\theta}}_{\ell}, \dot{\tilde{\beta}}_{0}=-\dot{\hat{\beta}}_{0}, \dot{\tilde{\beta}}_{m}=-\dot{\hat{\beta}}_{m}$, and using (1), (2), assumption A3, (16) with $i=m-1$, then one can compute the time derivative of (20) along the trajectories of $\dot{\mathbf{z}}_{m}$ and (18) as

$$
\begin{aligned}
\dot{V}_{m} \leq & -\sum_{j=1}^{m-1}\left(\varepsilon_{j}+\hat{\theta}_{j}\right)\left\|\mathbf{z}_{j}\right\|^{2}+\sum_{j=1}^{m} \theta_{j}\left\|\mathbf{z}_{j}\right\|^{2}+\mathbf{z}_{m-1}^{T} \mathbf{G}_{m-1} \mathbf{z}_{m} \\
& +\mathbf{z}_{m}^{T}\left[\mathbf{f}_{m}+\mathbf{G}_{m} \mathbf{u}+\mathbf{p}_{e s t}-\dot{\boldsymbol{\alpha}}_{(m-1) n}\right]+\beta_{0}\left\|\mathbf{z}_{m}\right\| \\
& -\sum_{\ell=1}^{m} \tilde{\theta}_{\ell} \dot{\hat{\theta}}_{\ell}+\beta_{m}\left\|\mathbf{z}_{m}\right\|\|\mathbf{x}\|-\tilde{\beta}_{0} \dot{\hat{\beta}}_{0}-\tilde{\beta}_{m} \dot{\hat{\beta}}_{m}
\end{aligned}
$$

Substituting the proposed controller (17) and adaptive gains (18) into the preceding equation for $\mathbf{z}_{m} \neq \mathbf{0}$ yields

$$
\begin{aligned}
\dot{V}_{m} \leq & -\sum_{j=1}^{m} \varepsilon_{j}\left\|\mathbf{z}_{j}\right\|^{2}+\beta_{m}\|\mathbf{x}\|\left\|\mathbf{z}_{m}\right\|-\left[\hat{\beta}_{0}+\hat{\beta}_{m}\|\mathbf{x}\|\right]\left\|\mathbf{z}_{m}\right\| \\
& +\beta_{0}\left\|\mathbf{z}_{m}\right\|-\left(\beta_{0}-\hat{\beta}_{0}\right) \dot{\hat{\beta}}_{0}-\left(\beta_{m}-\hat{\beta}_{m}\right) \dot{\hat{\beta}}_{m} \\
\leq & -\sum_{j=1}^{m} \varepsilon_{j}\left\|\mathbf{z}_{j}\right\|^{2} \leq 0 .
\end{aligned}
$$

It is easy to verify that $\dot{V}_{m} \leq-\sum_{j=1}^{m} \varepsilon_{j}\left\|\mathbf{z}_{j}\right\|^{2} \leq 0$ is also fulfilled for the case of $\mathbf{z}_{m}=\mathbf{0}$. Since $V_{m}>0$ and $\dot{V}_{m} \leq 0, V_{m}$ is a bounded function. From (20) and (13), it is known that $\mathbf{z}_{j}, \hat{\theta}_{j}, 1 \leq j \leq m, \hat{\beta}_{0}$, and $\hat{\beta}_{m}$, are all bounded. Equation (22) also clearly indicates that $\mathbf{z}_{j}$ will approach zero as $t \rightarrow \infty$. Then from (2), it can be seen that $\mathbf{x}_{1} \rightarrow \mathbf{0}$ as $t \rightarrow \infty$. According to (5) and (2), $\boldsymbol{\alpha}_{1}$ and $\mathbf{x}_{2}$ will reach zero as $t \rightarrow \infty$. Noted also that $\mathbf{x}_{1}$ and $\mathbf{x}_{2}$ are bounded since $\mathbf{z}_{1}, \mathbf{z}_{2}$, and $\alpha_{1}$ are bounded.

Suppose that $\overline{\mathbf{x}}_{\ell}, 1 \leq \ell \leq i$, and $\mathbf{z}_{j}, 1 \leq j \leq m$, all approach zero. Then according to the analysis in Appendix $\mathrm{C}$, one is able to see that the state variables $\mathbf{x}_{j}, 1 \leq j \leq m$, will be asymptotically stable.

(b) From (18), it is known that $\hat{\theta}_{j}(t), 1 \leq j \leq m, \hat{\beta}_{0}$, and $\hat{\beta}_{m}$ are monotonically increasing functions, and these functions are all bounded in accordance with (a). Therefore, according to the Proposition 2.14 in Tao [27], one can conclude that there exist finite constants $\theta_{j \infty}, 1 \leq j \leq m, \beta_{0 \infty}$, and $\beta_{m \infty}$ such that $\lim _{t \rightarrow \infty} \hat{\theta}_{j}(t)=\theta_{j \infty}, 1 \leq j \leq m, \lim _{t \rightarrow \infty} \hat{\beta}_{0}(t)=$ $\beta_{0 \infty}$, and $\lim _{t \rightarrow \infty} \hat{\beta}_{m}(t)=\beta_{m \infty}$ It is also noted that $\theta_{j \infty}$, $\beta_{0 \infty}$, and $\beta_{m \infty}$ may not necessarily be equal to $\theta_{j}, \beta_{0}$, and $\beta_{m}$ respectively, since $\dot{V}$ is a negative semidefinite function. (c) From (a), one can assume that $\overline{\mathbf{x}}_{\ell}, 1 \leq \ell \leq i$, and $\mathbf{z}_{j}$, $1 \leq j \leq m$, are all bounded. Then (30) implies that $\dot{\boldsymbol{\alpha}}_{(i-1) n}$ is bounded. From (15) it is seen that $\boldsymbol{\alpha}_{i}\left(\overline{\mathbf{x}}_{i}\right)$ is also bounded. Hence $\mathbf{x}_{i+1}$ is bounded in accordance with (2). Therefore from the mathematical induction point of view, one is able to conclude that $\mathbf{x}_{j}, 1 \leq j \leq m$, are all bounded. On the other hand, according to [26], the output of the derivative estimator $\mathbf{p}_{\text {est }}$ is also a bounded function due to the fact that $\mathbf{z}_{m}$ and $\overline{\mathbf{z}}_{m}$ are continuous. Therefore, the stability of overall controlled system is guaranteed due to the previous stability analysis.

Remark 3: Note that the unknown functions $\triangle \mathbf{f}_{i}(\bullet)$ may depend on the state variable $\mathbf{x}$, which means that if some terms in (1) do not satisfy the so called "Block StrictFeedback Form", then one can treat these terms as the uncertainty $\triangle \mathbf{f}_{i}(\mathbf{x})$. This is one of the advantages of the proposed control scheme. Noted also that these unknown functions $\triangle \mathbf{f}_{i}(\bullet)$ were all accumulated in the last step and were suppressed effectively by the adaptive gains $\hat{\theta}_{j}$.

\section{Numerical Example and Simulation}

Consider an MIMO system with dynamic equations in the form of (1), where $m=3$, the state variables are $\mathbf{x}_{1} \triangleq$ $\left[\begin{array}{ll}x_{11} & x_{12}\end{array}\right]^{T}, \mathbf{x}_{2} \triangleq\left[\begin{array}{ll}x_{21} & x_{22}\end{array}\right]^{T}, \mathbf{x}_{3} \triangleq\left[\begin{array}{lll}x_{31} & x_{32} & x_{33}\end{array}\right]^{T}$. The nonlinear vectors $\mathbf{f}_{i}$ and matrices $\mathbf{G}_{i}, 1 \leq i \leq 3$, are given by

$$
\begin{aligned}
\mathbf{f}_{1} & =\left[\begin{array}{c}
x_{11} \\
x_{11} x_{12}
\end{array}\right], \mathbf{f}_{2}=\left[\begin{array}{l}
x_{21}^{2} \\
x_{22}
\end{array}\right], \mathbf{G}_{1}=\left[\begin{array}{cc}
1 & 0 \\
x_{11} & 1
\end{array}\right] \\
\mathbf{f}_{3} & =\left[\begin{array}{c}
x_{11}+\sin \left(x_{31}\right) \\
x_{32} x_{22} \\
\sin \left(x_{33}\right)
\end{array}\right], \mathbf{G}_{2}=\left[\begin{array}{ccc}
1 & x_{22} & 0 \\
0 & 1 & 0
\end{array}\right], \\
\mathbf{G}_{3} & =\left[\begin{array}{ccc}
2+\sin \left(x_{31}\right) & 0 & 0 \\
0 & 2+\cos \left(x_{32}\right) & 0 \\
x_{11} x_{31} & 0 & 1
\end{array}\right] .
\end{aligned}
$$

The vector $\mathbf{u} \triangleq\left[\begin{array}{lll}u_{1} & u_{2} & u_{3}\end{array}\right]^{T}$ denotes the input. For demonstrating the robustness of the proposed control scheme using computer simulation, we assume that the unknown perturbations $\triangle \mathbf{f}_{i}, 1 \leq i \leq 3$, are

$$
\begin{aligned}
\Delta \mathbf{f}_{1} & =\left[\begin{array}{c}
0.1 x_{22}^{2}+2 \sin \left(x_{31}\right) \\
0.2 x_{11} \sin \left(x_{22}\right)+x_{22}
\end{array}\right], \\
\Delta \mathbf{f}_{2} & =\left[\begin{array}{c}
0.5 x_{32} \\
0.5 \sin \left(x_{31}\right)+6 x_{21} e^{-\frac{(t-2)^{2}}{3}}
\end{array}\right], \\
\triangle \mathbf{f}_{3} & =\left[\begin{array}{c}
x_{11} \sin \left(x_{31}\right)+0.2 x_{33}^{2} \sin (2 t) \\
0.2 x_{22}+0.2 x_{12} \cos (t) \\
0.5 \sin \left(x_{31} x_{21}\right)+0.1 x_{32}^{2}
\end{array}\right],
\end{aligned}
$$

respectively.

Noted that the preceding dynamic equations are not in block strict feedback form. The robust backstepping controller is designed in accordance with (17), and the designed parameters $\left(\varepsilon_{1}, \varepsilon_{2}, \eta, \beta_{0}, \beta_{1}, \beta_{2}, \beta_{3}\right)$ are all chosen to be 1 . The initial condition of each adaptive rule is 0 , and the unknown initial condition of $\mathbf{x}(t)$ are assumed to be $\mathbf{x}(0)=[-0.5,-0.5,1,1,0,0,-1]^{T}$. The results of computer simulation with step time $0.1 \mathrm{msec}$ are shown from Fig. 1 to Fig. 5. The state trajectories $\mathbf{x}(t)$, which are all driven to zero as $t \rightarrow \infty$, are given from Fig. 1 to Fig. 2 . The adaptive gains $\hat{\theta}_{j}, 1 \leq j \leq 3, \hat{\beta}_{0}$, and $\hat{\beta}_{3}$, which are all bounded and approach a constant respectively, are 


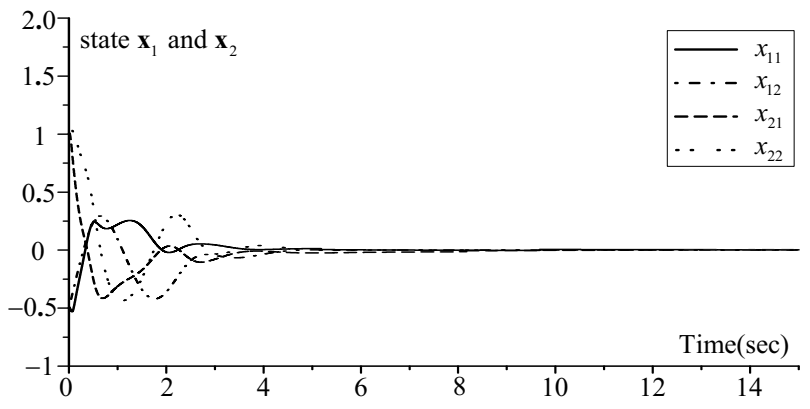

Fig. 1. Trajectories of state variables $\mathbf{x}_{1}$ and $\mathbf{x}_{2}$.

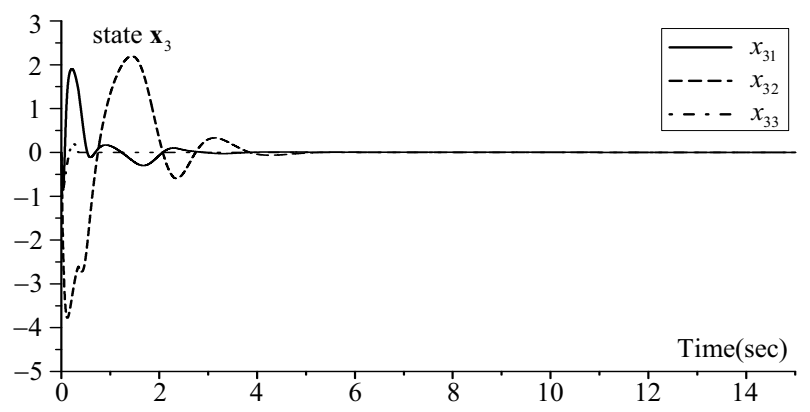

Fig. 2. Trajectories of state variable $\mathbf{x}_{3}$.

demonstrated in Fig. 3. Fig. 4 displays the perturbation estimation errors, which almost approach zero after $t>5$ sec. The designed perturbation estimator performs well even though there is an unexpected, large external disturbance " $6 x_{21} e^{-\frac{(t-2)^{2}}{3} "}$ occurring around the 2 nd sec in $\triangle \mathbf{f}_{2}$. Let the control energy consumed by the proposed control scheme be $E=\int_{0}^{t}\|\mathbf{u}\|^{2} d t$. It is clearly seen in Fig. 5 that the controlled system will consume a lot more energy if the controller contains no perturbation estimator at all.

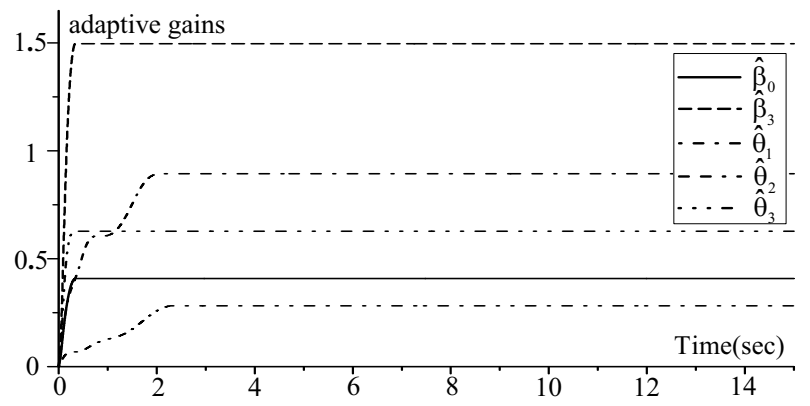

Fig. 3. Adaptive gains $\hat{\theta}_{j}, 1 \leq j \leq 3, \hat{\beta}_{0}$, and $\hat{\beta}_{3}$.

\section{Conclusions}

In this paper a robust adaptive backstepping control scheme with perturbation estimation embedded is successfully proposed for a class of perturbed multi-input nonlinear systems to solve regulation problems. The proposed method releases the restriction that the dynamic equations of controlled systems have to be in block strict feedback form, and it is also able to save control energy due to the usage of perturbation estimator. The adaptive mechanisms, which

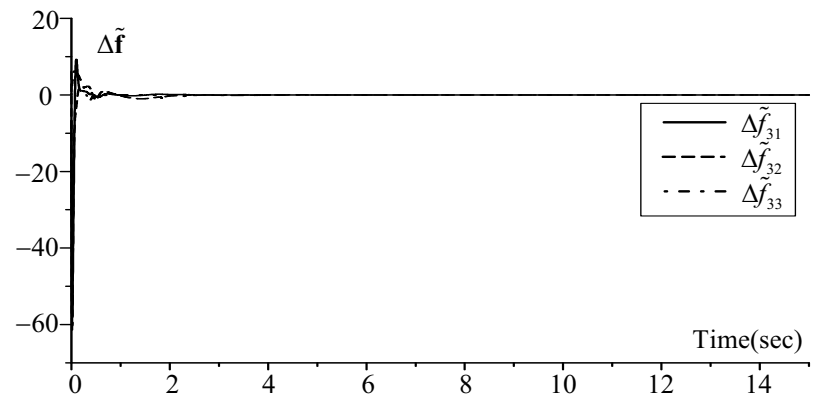

Fig. 4. Perturbations estimation errors $\Delta \tilde{\mathbf{f}}_{m}$.

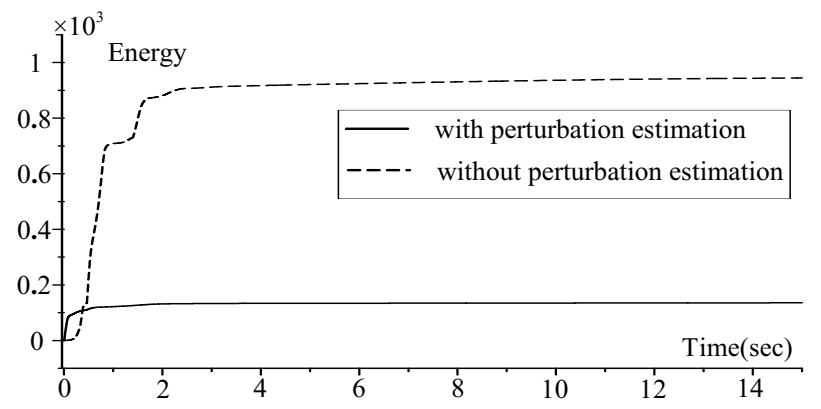

Fig. 5. Energy consumed by the controlled system with and without perturbations estimation.

are also embedded in the virtual inputs and the proposed controller, can effectively overcome the perturbations as well as estimation errors without knowing their least upper bounds in advance. However, the proposed control method is only able to alleviate, not totally eliminate, the disadvantage of "explosion of complexity". Therefore, for future study, designing adaptive block backstepping controllers without computing the derivatives of virtual input functions is worth considering.

\section{APPENDIX}

Appendix A: Consider the system (1) with the assumptions A1-A3. Suppose that the virtual inputs $\boldsymbol{\alpha}_{i}$ in the domain of interest satisfy the following constraints

$$
\left\|\boldsymbol{\alpha}_{i}\left(\overline{\mathbf{x}}_{i}\right)\right\| \leq \sum_{q=1}^{i} a_{i q}\left\|\mathbf{x}_{q}\right\|,
$$

where $a_{i q}, 1 \leq q \leq i$, are unknown positive constants. Then

$$
\left\|\triangle \mathbf{f}_{1}(\mathbf{x})\right\| \leq \sum_{s=1}^{m} r_{1 s}\left\|\mathbf{z}_{s}\right\|, \quad\left\|\mathbf{z}_{1}\right\|\left\|\triangle \mathbf{f}_{1}(\mathbf{x})\right\| \leq \sum_{\ell=1}^{m} \bar{r}_{1 \ell}\left\|\mathbf{z}_{\ell}\right\|^{2} .
$$

Proof: According to (2) and (24), the upper bounds of $\left\|\mathbf{x}_{i}\right\|$ is

$$
\left\|\mathbf{x}_{i}\right\| \leq\left\|\mathbf{z}_{i}\right\|+k_{i 1}\left\|\mathbf{z}_{1}\right\|+\cdots+k_{i(i-1)}\left\|\mathbf{z}_{i-1}\right\|
$$

where

$$
k_{i j} \triangleq\left\{\begin{array}{l}
\sum_{p=j}^{i-1} a_{(i-1) p} k_{p j}, \quad \text { if } i>j, \quad 1 \leq i \leq m \\
1, \quad \text { if } i=j
\end{array}\right.
$$


are unknown positive constants. From Assumption A3 and (26) one can compute the upper bound of $\left\|\triangle \mathbf{f}_{1}(\mathbf{x})\right\|$ and $\left\|\mathbf{z}_{1}\right\|\left\|\triangle \mathbf{f}_{1}(\mathbf{x})\right\|$ as (25), where $r_{1 s} \triangleq \sum_{q=s}^{m} b_{1 q} k_{q s}, 1 \leq s \leq$ $m, \bar{r}_{11} \triangleq r_{11}, \bar{r}_{1 \ell} \triangleq \frac{r_{1 \ell}}{2}, 2 \leq \ell \leq m$, are unknown positive constants.

Appendix B: Consider the system (1) with the assumptions A1-A3 and (24). Suppose that the virtual inputs $\dot{\alpha}_{i p}$ in the domain of interest satisfy the following constraints

$$
\left\|\dot{\boldsymbol{\alpha}}_{i p}(\mathbf{x})\right\| \leq \sum_{\ell=1}^{m} d_{i \ell}\left\|\mathbf{x}_{\ell}\right\|,
$$

where $d_{i \ell}, 1 \leq \ell \leq m, 1 \leq i \leq m-1$, are unknown positive constants. Then

$$
\begin{aligned}
&\left\|\triangle \mathbf{f}_{i}(\mathbf{x})-\dot{\alpha}_{(i-1) p}\right\| \leq \sum_{s=1}^{m} r_{i s}\left\|\mathbf{z}_{s}\right\|, \quad \text { and } \\
&\left\|\mathbf{z}_{i}\right\|\left\|\triangle \mathbf{f}_{i}(\mathbf{x})-\dot{\alpha}_{(i-1) p}\right\| \leq \sum_{\ell=1}^{m} \bar{r}_{i \ell}\left\|\mathbf{z}_{\ell}\right\|^{2} .
\end{aligned}
$$

Proof: From Assumption A3, (26), (27), and (2), one can compute the upper bounds of $\left\|\triangle \mathbf{f}_{i}(\mathbf{x})-\dot{\alpha}_{(i-1) p}\right\|$ and $\left\|\mathbf{z}_{i}\right\|\left\|\triangle \mathbf{f}_{i}(\mathbf{x})-\dot{\alpha}_{(i-1) p}\right\|$ as (28), (29) respectively; where $r_{i s} \triangleq \bar{b}_{i s}+\bar{d}_{(i-1) s}, 1 \leq s \leq m, \bar{r}_{i i} \triangleq r_{i i}, \bar{r}_{i \ell} \triangleq \frac{r_{i \ell}}{2}$, $1 \leq \ell \leq m, \ell \neq i$, are unknown positive constants.

Appendix C: According to (15) and (1), one can compute the derivative of $\boldsymbol{\alpha}_{i-1}$ with respect to time as

$$
\frac{d \boldsymbol{\alpha}_{i-1}}{d t} \triangleq \dot{\alpha}_{(i-1) n}\left(\overline{\mathbf{x}}_{i}\right)+\dot{\alpha}_{(i-1) p}(\mathbf{x})
$$

where

$$
\begin{aligned}
\dot{\boldsymbol{\alpha}}_{(i-1) n}\left(\overline{\mathbf{x}}_{i}\right)= & \sum_{l=1}^{i-1} \frac{\partial \boldsymbol{\alpha}_{i-1}}{\partial \mathbf{x}_{l}}\left[\mathbf{f}_{l}\left(t, \overline{\mathbf{x}}_{l}\right)+\mathbf{G}_{l}\left(\overline{\mathbf{x}}_{l}\right) \mathbf{x}_{l+1}\right] \\
& +\sum_{l=1}^{i-1} \frac{\partial \boldsymbol{\alpha}_{i-1}}{\partial \hat{\theta}_{l}}\left\|\mathbf{z}_{l}\right\|^{2} \\
\dot{\boldsymbol{\alpha}}_{(i-1) p}(\mathbf{x})= & \sum_{l=1}^{i-1} \frac{\partial \boldsymbol{\alpha}_{i-1}}{\partial \mathbf{x}_{l}} \triangle \mathbf{f}_{l}(t, \mathbf{x}) .
\end{aligned}
$$

Suppose that $\overline{\mathbf{x}}_{\ell}, 1 \leq \ell \leq i$, and $\mathbf{z}_{j}, 1 \leq j \leq m$, all approach zero. Then (30) implies that $\dot{\alpha}_{(i-1) n}$ converges to zero as $t \rightarrow \infty$. From (15) it is seen that $\boldsymbol{\alpha}_{i}\left(\overline{\mathbf{x}}_{i}\right)$ will also approach zero as $t \rightarrow \infty$. Hence $\mathbf{x}_{i+1} \rightarrow \mathbf{0}$ as $t \rightarrow \infty$ in accordance with (2). Therefore from the mathematical induction point of view, one is able to conclude that $\mathbf{x}_{j}$, $1 \leq j \leq m$, will be asymptotically stable.

\section{REFERENCES}

[1] J. Y. Hung, W. Gao, and J. C. Hung, Variable structure control: a survey, IEEE Trans. Ind. Electron., vol. 40, no. 1, pp. 2-22, 1993.

[2] E. Christopher and S. K. Spurgeon, Sliding Mode Control, Theory and Applications, New York: Taylor \& Francis, 1998.

[3] C. C. Cheng, C. C. Wen, and W. T. Lee, Design of decentralised sliding surfaces for a class of large-scale systems with mismatched perturbations, Int. J. Control, vol. 82, no. 11, pp. 2013-2025, 2009.

[4] Y. Chang and C. C. Cheng, Adaptive sliding mode control for plant with mismatched perturbations to achieve asymptotical stability, Int. J. Robust. Nonlinear Control, vol. 17, no. 9, pp. 880-896, 2007.
[5] C. C. Cheng and Y. Chang, Design of decentralised adaptive sliding mode controllers for large-scale systems with mismatched perturbations, Int. J. Control, vol. 81, no. 10, pp. 1507-1518, 2008.

[6] X.-G. Yan, S. K. Spurgeon, and C. Edwards, Global stabilisation for a class of nonlinear time-delay systems based on dynamical output feedback sliding mode control, Int. J. Control, vol. 82, no. 12, pp. 2293-2303, 2009

[7] H. K. Khalil, Nonlinear Control, New Jersey: Prentice-Hall, 1996.

[8] M. Krstic, I. Kanellakopoulos, and P. Kokotovic, Nonlinear and Adaptive Control Design, New York: John Wiley \& Sons, Inc. 1995.

[9] Y. Zhang, S. Li, and Q. Zhu, Backstepping-enhanced decentralised PID control for MIMO processes with an experimental study, IET Control Theory \& Applic., vol. 1, no. 3, pp. 704-712, 2007.

[10] J. Fu, Extended backstepping approach for a class of nonlinear systems in generalised output feedback canonical form, IET Control Theory \& Applic., vol. 3, no. 8, pp. 1023-1032, 2008.

[11] C. Hua, G. Feng, and X. Guan, Robust controller design of a class of nonlinear time delay systems via backstepping method, Automatica, vol. 44 , no. 2, pp. 567-573, 2008.

[12] B. Chen, X. Lin, K. Lin, and C. Lin, Novel adaptive neural control design for nonlinear systems, Automatica, vol. 45, pp. 1554-1560, 2009.

[13] J. T. Huang, Hybrid-based adaptive NN backstepping control of strictfeedback systems, Automatica, vol. 45 pp. no. 6, 1497-1503, 2009.

[14] B. Yao and M. Tomizuka, Adaptive robust control of multi-input nonlinear systems in semi-strict feedback forms, Automatica, vol. 37, no. 7, pp. 1305-1321, 2001.

[15] A. J. Koshkouei, A. S. I. Zinober, and K. J. Burnham, Adaptive sliding mode backstepping control of nonlinear systems with unmatched uncertainty, Asian J. of Control, vol. 6, no. 4, pp. 447-453, 2004.

[16] M. Wang, B. Chen, X. Liu, and P. Shi, Adaptive fuzzy tracking control for a class of perturbed strict feedback nonlinear time-delay systems, Fuzzy Sets and Syst., vol. 159, no. 8, pp. 949-967, 2008.

[17] M. Wang, B. Chen, and S. Zhang, Adaptive neural tracking control of nonlinear time-delay systems with disturbances, Int. J. Adapt. Control Signal Process., vol. 23, no. 11, pp. 1031-1049, 2009.

[18] Y. Chang and C. C. Cheng, Block backstepping control of multiinput nonlinear systems with Mismatched Perturbations for asymptotic stability, Int. J. Control, vol. 83, no. 10, pp. 2028-2039, 2010.

[19] Y. Chang, Block backstepping control of MIMO systems, IEEE Trans. Autom. Control, vol. 56, pp. 1191-1197, 2011.

[20] C.-C. Cheng, G.-L. Su, and C.-W. Chien, Block backstepping controllers design for a class of perturbed nonlinear systems with $\mathrm{m}$ blocks, IET Control Theory \& Applic., accepted for publication.

[21] C. C. Cheng, Y.-S. Lin, and A.-F. Chien, Design of backstepping controllers for systems with non-strict feedback form and application to chaotic synchronization, Proc. 18th IFAC World Congr., Milano, Italy, pp. 10964-10969, Aug. 2011.

[22] Y. Li, S. Tong, Y. Li, Observer-based adaptive fuzzy backstepping control of MIMO stochastic nonlinear strict-feedback systems, Nonlinear Dyn., vol. 67, pp. 1579V1593, 2012.

[23] W. S. Chen, Adaptive backstepping dynamics surface control for systems with periodic disturbances using neural networks, IET Control Theory \& Applic., vol. 3, no. 10, pp. 1383-1394, 2009.

[24] S. J. Yoo, J. B. Park, Neural-network-based decentralized adaptive control for a class of large-scale nonlinear systems with unknown time-varying delays, IEEE Trans. Syst., Man, Cybern. B, Cybern., vol. 39, no. 5, pp. 1316-1323, 2009.

[25] C. C. Cheng, J. M. Hsiao, and Y. P. Lee, Design of robust tracking controllers using sliding mode technique, JSME Int. J. Series C, Mechanical Systems, Machine Elements and Manufacturing, vol. 44, pp.89-95, 2001.

[26] C. C. Cheng and M. W. Chang, Design of derivative estimator using adaptive sliding mode technique, Proc. of 2006 American Control Conf., Minneapolis, Minnesota USA, pp. 2611-2615, June 2006.

[27] Tao, G., Adaptive Control Design and Analysis, John Wiley \& Sons, Inc. New York, 2003. 